\title{
The Experiment of the Clog Reduction in a Plane Silo
}

\author{
Ai-Le Sun ${ }^{1}$ and Jie Zhang ${ }^{1,2, \star}$ \\ ${ }^{1}$ Department of Physics and Astronomy, Shanghai Jiao Tong University, Shanghai, China 200240. \\ ${ }^{2}$ Institute of Natural Sciences, Shanghai Jiao Tong University, Shanghai, China 200240.
}

\begin{abstract}
The flow of particles may be clogged when they pass through a narrow orifice. Many factors can change the probability of clogging, such as the outlet size, the presence of obstacles and external perturbation, but the detailed mechanisms are still unclear. In this paper, we present an experimental study of reduction of the clogging probability in a horizontal plane silo, which consists of a layer of elastic particles transported on an annular flat plate rotating with a constant angular velocity passing through a hopper structure. We found the exponential distributions of the avalanche size for different sizes of orifice and the power law tails of the passing time between two particles. We did not confirm whether there was a critical size of orifice above which the clogging became impossible. We explored the effect of the obstacle on the probability of clogging: and if we chose a proper obstacle placed at a proper position, the probability of clogging could be reduced by a factor of about seven.
\end{abstract}

\section{Introduction}

Granular materials are very common in our daily life, such as sand, powder, cement, where the motions of particles due to thermal fluctuations are negligible[1,2]. When lots of granular particles pass through a small orifice, the flow of particles could be abruptly arrested, i.e. clogged. This phenomenon is ubiquitous and has be observed in many systems, such as granular flows in a hopper [3-7], dense suspension flows in a micro-channel [8], emulsions [9, 10], dense colloidal systems [11], and living animals [12]. In addition, when in an emergency, people rush to escape through a narrow exit, the clog may appear [13].

Many factors can affect clogging, such as the size of orifice, presence of an obstacle, and external vibrations. Previous studies have revealed the exponential distribution of avalanche size. To and colleagues [3] proposed a restricted random walk model to explain the exponential distribution by analyzing the arches of particles. This model provides a good fitting to the data. However, the motion of particle is not necessary like a restricted random walk and the friction between the particles is large in many systems. Another problem is whether there is a critical outlet size that the probability of clog decreases to zero. CC.Thomas[14] proposed that the critical outlet size does not exist. To further study this problem, we designed a novel apparatus where the particles can be automatically refilled so that the experiment can run continuously and particles in the hopper will never be exhausted. In addition, one interesting thing is that if an obstacle is placed at a proper position, the silo will have much less chance to $\operatorname{clog}[4,15]$. In this paper, we will also present some pre-

\footnotetext{
^e-mail: jiezhang2012@sjtu.edu.cn
}

liminary results related to the reduction of clogging by an obstacle.

\section{Experimental Setup}

In order to probe the key factors that influence the probability of clogging, we designed a $2 \mathrm{D}$ annular silo that allowed the particles to be recycled, so that the experiment could run continuously. The silo was filled with elastic particles, which were bidisperse. The Young's modulus of particles is $6 \mathrm{MPa}$. The total number of particles was $\sim 1400$, including $\sim 600$ large particles of a diameter of $14 \mathrm{~mm}$ and $\sim 800$ small particles of a diameter of $12 \mathrm{~mm}$ in order to avoid crystallization. [16]

As shown in Fig. 1, the radius of the large circle was $R_{2}=0.5 \mathrm{~m}$ and the radius of the small circle was $R_{1}=0.1 \mathrm{~m}$. The particles were driven by the frictional force between the particles and the bottom plate that was rotating at a constant angular velocity of $\omega=\frac{\pi}{45} \mathrm{rad} / \mathrm{sec}$. There were two Basler cameras mounted above the apparatus to record the motion of particles, and the internal stress information of particles. When the silo was clogged for a sufficiently long time, we would apply a manual perturbation to break the arch formed near the orifice by turning on a small vibrator. One important control parameter of this system is the size of the orifice in mean particle diameters. We systematically changed the size of orifice and the position of obstacle. Through the imaging analysis, we extracted the location of particles in the system, and used the result to track the motion of the particles. 


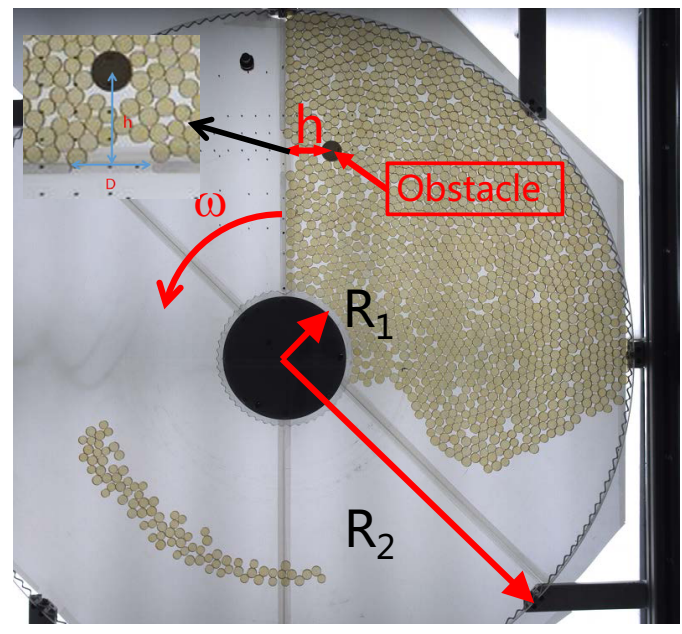

Figure 1. The experimental setup. The apparatus is essentially a horizontal silo, which consists of an annular plate of a large radius of $50 \mathrm{~cm}$ and a small radius of $10 \mathrm{~cm}$. Particles were driven by the frictional forces between the particles and the plate, which rotated counter-clockwise with an angular velocity of $\frac{\pi}{45} \mathrm{rad} / \mathrm{s}$. A hopper structure is visible on the plot along with an obstacle (the black blob on the figure).Particles clog the orifice intermittently.

\section{Results}

\subsection{The time lapse of particles}

To define a clog, we need to analyze the interval time between two consecutive particles passing through the orifice. What will be the proper interval time for us to affirm that the silo is clogged? The data were obtained by analyzing images of particles passing through the orifice. The flow could be intermittent. Figure 2(a) plots the survival or reliability function of the interval time between two consecutive particles passing through the orifice. We found that the survival had a power law tail, which is consistent with the results of Zuriguel et al.[17]. The difference is that in their experiment the sheep could adjust themselves to get out of the exit if a clog happened, but in particulate system the particles can not adjust themselves unless a perturbation is applied.

Based on the above results, we can choose a proper critical interval of time $\Delta t_{c}$ to define the clog. As Fig. 2(b) shows, we can choose different $\Delta t_{c}$ and the distribution of avalanche size remains almost the same. Here the $\mathrm{x}$ axis is normalized by the average avalanche size, and the y axis is the probability of the corresponding avalanche size (the average of frequency). When $\Delta t_{c}$ is large, the distribution is exponential, and only when $\Delta t_{c}$ is sufficiently small, i.e. $\Delta t_{c}=0.25 \mathrm{~s}$, the distribution becomes different. As we mentioned before we would apply perturbations to break permanent clog in order to perform a new measurement. The frequency of $\Delta t>10 \mathrm{~s}$ is rather small and we can classify them in one bin. That means the choice of $\Delta t_{c}$ does not strongly influence the results of statistics. So we simply chose the $\Delta t_{c}=10 \mathrm{~s}$ as the critical time in our later experiments.

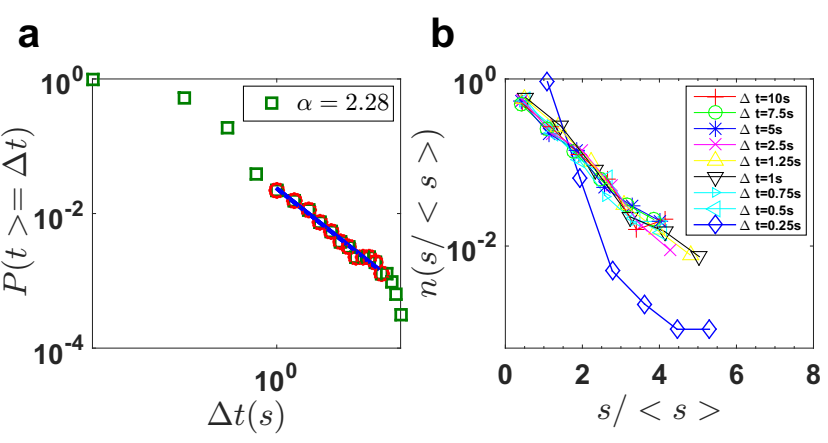

Figure 2. (a)The cumulative probability distribution function versus time interval $\Delta t$. (b) The probability distributions of avalanche size $s$ normalized by its mean $\langle s\rangle$ for a set of different time intervals $\Delta t$.

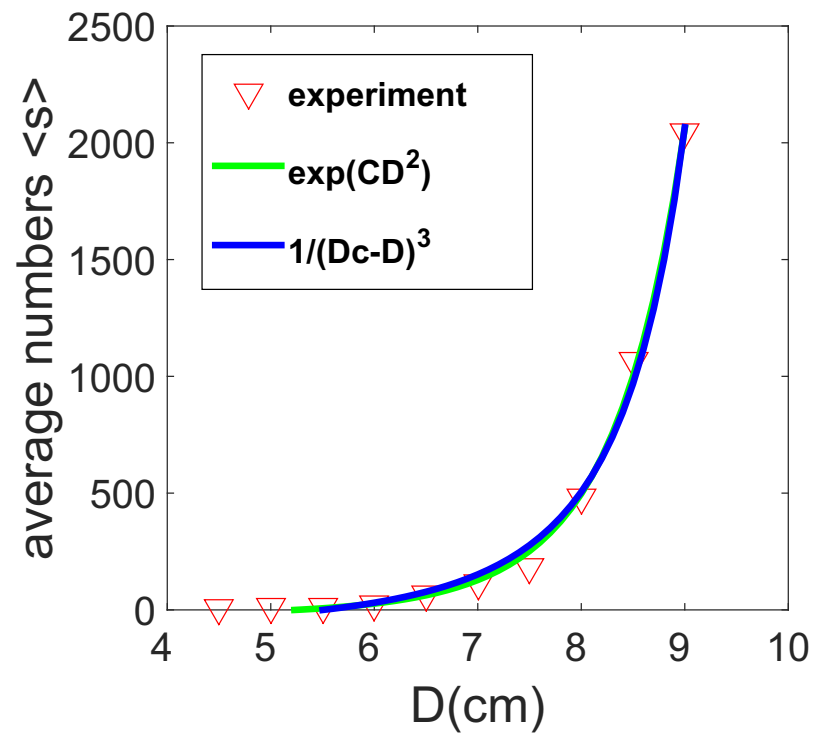

Figure 3. The mean avalanche size $\langle s\rangle$ versus the size of outlet. An exponential fitting(green) and a power law fitting(blue) can fit the data equally well.

\subsection{The effect of outlet size}

We found the avalanche size was an exponential distribution, consistent with the early finding of To et al. [3]. The model assumes that the probability of one particle passes through the outlet as a constant, denoted as $\mathrm{p}$, so the probability of passing through $\mathrm{n}$ particles and then the flow being stopped can be written as:

$$
p(n)=p^{n}(1-p)
$$

So the average size of an avalanche is

$$
<n>=\frac{\sum n * p(n)}{\sum p(n)}
$$

We measured the avalanche sizes for 10 different outlet sizes by fixing the angular velocity. we selected the 
critical time interval $\Delta t_{c}=10 \mathrm{~s}$ to define the clog. Every point is an average of nearly 150 avalanches. As Fig. 3(b) shows, we can see the influence of the outlet size. When the diameter of outlet enlarged, the average avalanche size increased as expected. When the outlet size exceeded $8 \mathrm{~cm}$, the clog happened rarely. In our experiment, the flow continued for a long time, but the clog still happened after a sufficiently long time. That means if the flow was clogged, and sometimes the arch of particles was very stable unless we applied a perturbation to the system. This is much more difficult to be observed in systems of finite size because it needs to refill particles into the system once the particles are empty.

\subsubsection{Critical outlet}

The problem is whether there is a critical outlet size that the flow can never be stopped. As we have mentioned early that in our system the probability of clog decreased as the outlet size increased. we fit our data by two means: (1)an exponential fitting and (2)a power law fitting.

First, we applied exponential function to fit the data. In $2 \mathrm{D}$ systems, the average size of an avalanche is proportional to $\exp \left(C D^{2}\right),[14](\mathrm{C}$ is a constant related to the system.)(in 3 dimension is $\exp \left(C D^{3}\right)$.) In addition, we used a power law to fit the data. The hypothesis is that there is a parameter, named $D_{c}$, which is the critical outlet size and the average avalanche size is divergent. From the figure, we can see that both descriptions are comparable in fitting the experimental results.

If the power law were better, there would exist a critical outlet size and if the exponent fitting were better, there would not exist a critical outlet size. Based on the fitting, we calculated the critical outlet size to be around $10.8 \mathrm{~cm}$. Now the difficulty is that if $\mathrm{D}$ is larger than $9.5 \mathrm{~cm}$, we could hardly observe the clogging, although our system can be automatically refilled, the interval between twice clog is increasing sharply and exceed our observation time .Thus we did not confirm whether there was a critical size of orifice above which the clogging became impossible.

\subsection{The effect of the height of obstacle}

The average avalanche size will increase if a proper obstacle is put at an appropriate position. $[4,15]$ The obstacle can influence the clogging process significantly. The shape and size of obstacle play significant roles here.

In order to probe the influence of obstacle, we measured 11 different positions for a fixed obstacle size and the outlet size. We found that the exponential distribution is still valid with the presence of an obstacle. It is reasonable to assume that the probability of clog without an obstacle equals the one when the obstacle is far from the outlet. As Fig. 4 shows(when $D=5.5 \mathrm{~cm}$ ), when the distance of obstacle relative to the outlet was larger than $9 \mathrm{~cm}$, the influence of obstacle could be neglected. When the distance decreased to about $5 \mathrm{~cm}$, the average avalanche size had a maximum value, about three times of that without an obstacle. When the distance decreased below $5 \mathrm{~cm}$,

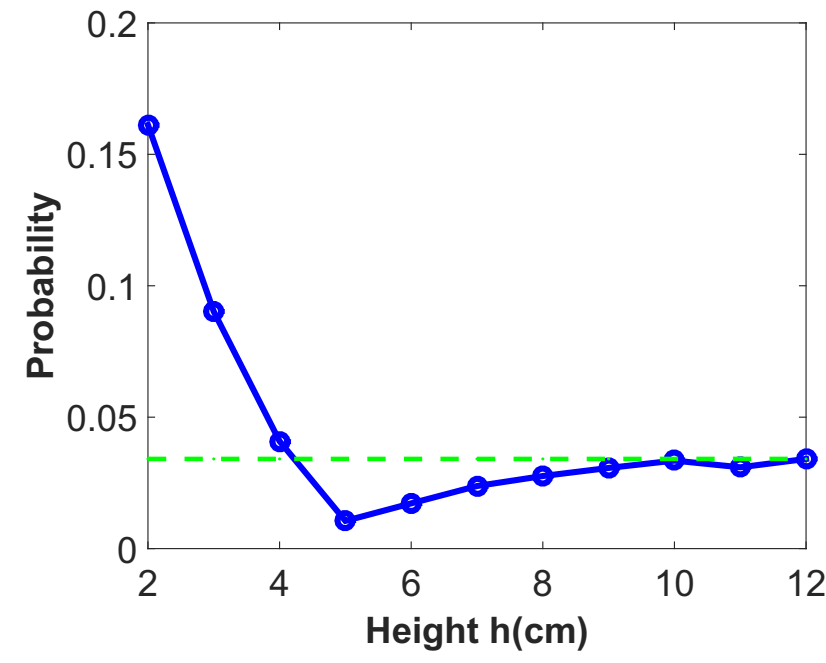

Figure 4. The probability of clogging versus the height $h$ of obstacle that is defined in Fig. 1.The dash line is the probability of no obstacle.

the probability of clog would increase and even exceed the probability without an obstacle. The main reason is when the obstacle was close to the orifice, the obstacle stopped flow of the particles, so that the particles could only form arches at the two sides of the obstacle rather than the front of obstacle. In conclusion, if we choose proper obstacle and put it on an appropriate position, the probability can be diminished by a factor of 3 .

The reason of reducing clog by an obstacle is still controversial but we tend to agree with the idea that the obstacle relieves the pressure of particles. The obvious parameter that influences the clog is the position of obstacle. The main zone is the front of the obstacle, so if the zone is sufficiently larger the pressure can be supported by the obstacle, the probability of clog will decrease. In our experiment, when $\mathrm{h}>5 \mathrm{~cm}$, particle could form the arches at the orifice, so the pressure would decrease and the force chain was relatively weak. In addition, the regime was relatively large, so that the arches were usually unstable and could be destroyed by small perturbations of particles coming from the upstream flow. Therefore the probability of clog was small. When $\mathrm{h}$ was too large, the shield of the obstacle would become weaken gradually until the height reached infinite, the effect of obstacle would vanish completely.

Note that the factor of the clog reduction probability was about 3 , smaller than the previous research. We changed the diameter of the obstacle, and measured the average avalanche size at $\mathrm{h}=5 \mathrm{~cm}$. As a result, we found the ratio became larger, about 7 . Limited by our system, we could not use a bigger obstacle. In addition, we think the variety of particle is the main difference from others' work. So we will use other types of particles in future. 


\section{Conclusion}

In this paper, we presented an experimental study of reduction of the probability in a plane silo by changing the ratio of outlet vs particles by the presence of an obstacle. The setup consisted of a layer of elastic particles transported on an annular flat plate of a constant angular velocity passing through a hopper structure. By tracking the motion of particles we get an analysis of the reduction of clogging in a two dimensional plane silo.

In this experiment, we placed an obstacle at different locations near the exit of the silo in order to investigate the influence of the obstacle to the clogging process. We found that a system driven by frictional force is quite different from a system driven by gravity. Since the particles were recycled in the system, we could explore the evolution of the clogging process in an infinitely long time scale, in principle. We measured the time between two consecutive particles which passed through the outlet and found the time distribution had a power law tail.

We studied the influence of the outlet size and the position of obstacle. By fitting the data, we found an exponential fitting and a power law fitting are comparable in describing the statistics of clogging. Now there is a debate about whether there is a critical outlet size above which the clogging is impossible. Based on our experiment data we couldn't confirm whether there is a critical outlet size. We think when the outlet size becomes bigger, the probability of clogging will become smaller until we can not obverse the clog, therefore it is hard to prove in experiment.

Then we put an obstacle on the front of the outlet and found the probability of clogging had been changed significantly. In our experiment, the probability of clogging could be reduced by a factor of 7 or more. The reduction of the probability of clogging is possibly caused by the reduction of the pressure at the down stream of the obstacle, and we will explore it in our future work. The size of the obstacle has a significant influence on the clog. If we choose proper obstacle, the factor may become bigger.

\section{Acknowledgements}

J.Z. acknowledges support from the award of the Chinese 1000-Plan (C) fellowship and National Natural Science Foundation of China (11474196).

\section{References}

[1] H.M. Jaeger, S.R. Nagel, R.P. Behringer, Rev. Mod. Phys. 68, 1259 (1996)

[2] P.G. de Gennes, Reviews of modern physics 71, S374 (1999)

[3] K. To, P.Y. Lai, H. Pak, Physical review letters 86, 71 (2001)

[4] I. Zuriguel, A. Janda, A. Garcimartín, C. Lozano, R. Arévalo, D. Maza, Physical review letters 107, 278001 (2011)

[5] D.J. Durian, Phys. Rev. Lett. 75, 4780 (1995)

[6] I. Zuriguel, T. Mullin, J.M. Rotter, Phys. Rev. Lett. 98, 028001 (2007)

[7] J. Tang, R.P. Behringer, EPL (Europhysics Letters) 114, 34002 (2016)

[8] K.L. Rock, C. Gramm, L. Rothstein, K. Clark, R. Stein, L. Dick, D. Hwang, A.L. Goldberg, Cell 78, 761 (1994)

[9] X. Hong, K.W. Desmond, D. Chen, E.R. Weeks, arXiv preprint arXiv:1503.07569 (2015)

[10] X. Hong, M. Kohne, E.R. Weeks, arXiv preprint arXiv:1512.02500 (2015)

[11] C.P. Ortiz, R. Riehn, K.E. Daniels, Soft Matter 9, 543 (2013)

[12] I. Zuriguel, D.R. Parisi, R.C. Hidalgo, C. Lozano, A. Janda, P.A. Gago, J.P. Peralta, L.M. Ferrer, L.A. Pugnaloni, E. Clément et al., Scientific reports 4 (2014)

[13] I. Zuriguel, Papers in physics 6, 0 (2014)

[14] C. Thomas, D. Durian, Physical review letters 114, 178001 (2015)

[15] C. Lozano, A. Janda, A. Garcimartín, D. Maza, I. Zuriguel, Physical Review E 86, 031306 (2012)

[16] D. Helbing, A. Johansson, J. Mathiesen, M.H. Jensen, A. Hansen, Physical review letters 97, 168001 (2006)

[17] A. Garcimartín, J. Pastor, L. Ferrer, J. Ramos, C. Martín-Gómez, I. Zuriguel, Physical Review E 91, 022808 (2015) 